\title{
HIT BY HEPARIN: A Rare Case of Late Onset Heparin Induced Thrombocytopenia
}

\author{
HAMZA HASHMI MD*, UMAYR AZIMI MD \\ Grand Rapids Medical Education Partners \\ *Corresponding author: hamza.hashmi@grmep.com \\ Received April 27, 2015; Revised May 09, 2015; Accepted May 19, 2015
}

\begin{abstract}
Heparin induced thrombocytopenia is a rare complication of heparin therapy that usually occurs within initial 5 to 10 days of heparin exposure. We present a rare case of delayed onset heparin induced thrombocytopenia that occurred one week after cessation.
\end{abstract}

Keywords: heparin induced thrombocytopenia, argatroban, coronary artery bypass grafting, serotonin release assay

Cite This Article: HAMZA HASHMI MD, and UMAYR AZIMI MD, "HIT BY HEPARIN: A Rare Case of Late Onset Heparin Induced Thrombocytopenia.” American Journal of Medical Case Reports, vol. 3, no. 6 (2015): 174-176. doi: 10.12691/ajmcr-3-6-7.

\section{Introduction}

Heparin induced thrombocytopenia is a rare and potentially life threatening complication of heparin therapy. There is a temporal relationship between heparin exposure and thrombocytopenia. It usually happens within initial 5 to 10 days of heparin exposure and involves arterial and venous thromboembolic events. In rare instances, it might occur 2 to 3 weeks after cessation of heparin therapy [1]. We present a rare case of delayed onset heparin induced thrombocytopenia that occurred 17 days after cessation of heparin therapy and resulted in significant thromboembolic events.

\section{Patient Description}

A 65 year old male patient with past medical history significant for type 2 diabetes mellitus and hypertension presented to the emergency department of our hospital with the chief complaint of sudden onset shortness of breath of several hours duration. EKG revealed ST segment depressions in leads II, III and aVF. Troponin I was found to be elevated at $3.2 \mathrm{mcg} / \mathrm{L}$. Chest $\mathrm{X}$ ray did not reveal any acute cardiac or pulmonary process. CT angiogram of the chest was negative for pulmonary embolism. Elevated troponins and EKG changes raised concerns for acute coronary syndrome. Patient was given a bolus of IV unfractionated heparin and started on a continuous drip. He had a transthoracic echocardiogram done which revealed diminished ejection fraction of $30 \%$ with inferior and lateral wall motion abnormalities. In the meantime his shortness of breath improved and he was weaned off oxygen. On day 1 of admission he underwent cardiac catheterization which revealed severe triple vessel disease. On day 3 of admission patient underwent coronary artery bypass grafting with one left internal mammary artery graft and two saphenous vein grafts. Pre procedure IV heparin was discontinued after total of 48 hours of therapy. Patient had an uneventful hospital course and was eventually discharged home after 10 days of hospital stay. Upon discharge he had stable vital signs, normal platelet count of $170 \times 10^{9} / \mathrm{L}$ and improved ejection fraction of $40 \%$ on transthoracic echocardiogram.

Ten days after discharge he presented to the emergency department again with gradually worsening right lower extremity swelling of three days duration. The leg swelling was associated with exertional dyspnea and intermittent right sided chest pain. Upon examination he was found to be hypoxic with oxygen saturation of $85 \%$ on room air. A right lower extremity Doppler ultrasound was obtained and revealed deep vein thrombosis involving the right sided femoral vein. He was started on IV unfractionated heparin after intravenous bolus. Complete blood count revealed thrombocytopenia with a platelet count of $80 \times 109$ / L. Given hypoxia CT angiogram of the chest and transthoracic echocardiogram were obtained and revealed right sided subsegmental pulmonary artery thrombus and a small left ventricular thrombus respectively. Given constellation of thrombocytopenia, recent heparin exposure and venous as well as arterial thrombotic events concern for heparin induced thrombocytopenia was raised. His $4 \mathrm{~T}$ score was found to be 6 which indicated high probability of heparin induced thrombocytopenia. On Day 2 of admission and after 24 hours of continuous infusion, heparin drip was stopped and argatroban infusion was started without a bolus. ELISA for anti-PF4 antibodies was elevated at 3.0 OD and serotonin release assay also came back strongly positive. As platelet count normalized on day 5 of admission warfarin was started. Patient was bridged with argatroban and warfarin for 5 days. Once the INR was therapeutic (2-3) for more than 24 hours he was 
discharged on warfarin for 6 months. Follow up visit at 3 and 6 months interval revealed normal platelet count and

A.

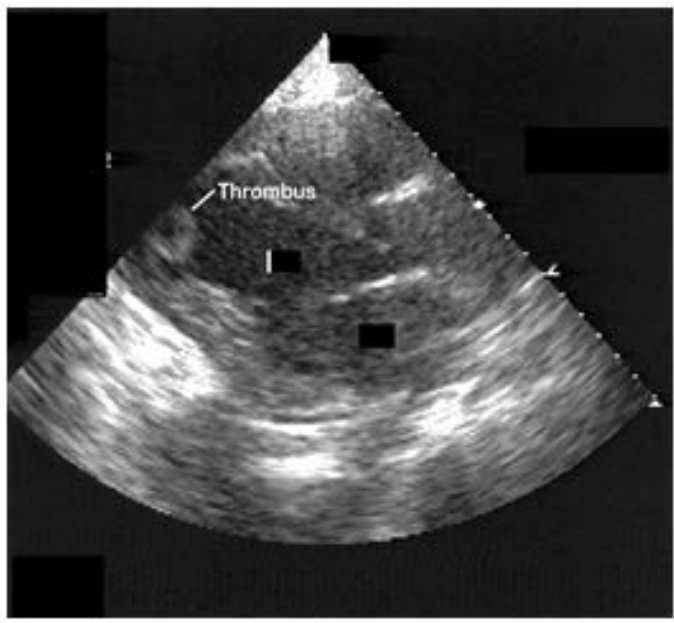

complete resolution of lower extremity DVT and left ventricular thrombus.

B.

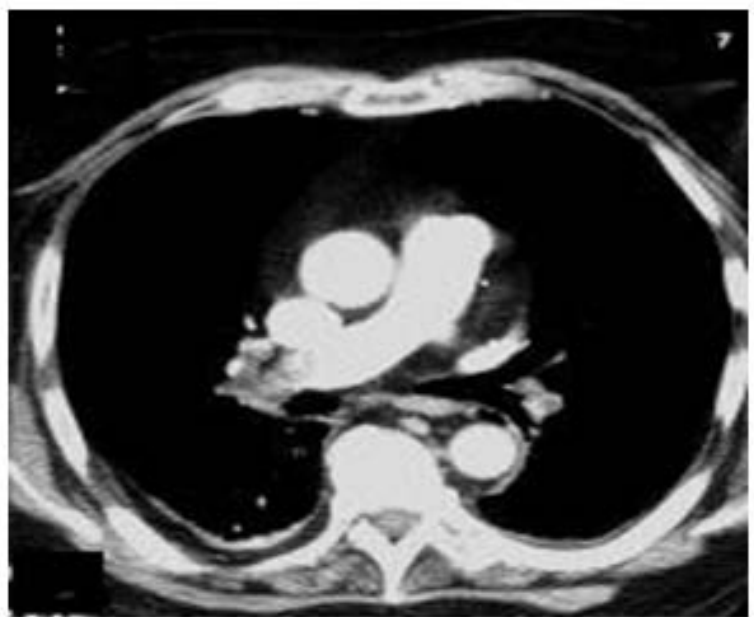

Figure 1. A. Deep vein thrombosis in the right femoral vein B. Pulmonary embolus in in the subsegmental branchof right pulmonary artery in a patient developing Heparin Induced Thrombocytopenia 14 days after last exposure to heparin (Delayed onset HIT)

Table 1. Represents important events with corresponding platelet counts

\begin{tabular}{|c|c|c|}
\hline $\begin{array}{c}\text { DAY } \\
\text { (Since initial } \\
\text { presentation) }\end{array}$ & $\begin{array}{c}\text { PALTELET } \\
\text { COUNT } \\
\text { (x 109 / L) }\end{array}$ & SIGNIFICANTEVENT \\
\hline 0 & 210 & $\begin{array}{c}\text { Initial Heparin bolus and } \\
\text { infusion }\end{array}$ \\
\hline 3 & 180 & $\begin{array}{c}\text { CABG and cessation of heparin } \\
\text { therapy }\end{array}$ \\
\hline 10 & 200 & \begin{tabular}{c} 
Discharge \\
\hline 17
\end{tabular} \\
\hline 18 & 70 & $\begin{array}{c}\text { Readmission with DVT, } \\
\text { Pulmonary embolism and left } \\
\text { ventricular thrombus }\end{array}$ \\
\hline 22 & 160 & $\begin{array}{c}\text { Cessation of heparin } \\
\text { Initiation of argatroban }\end{array}$ \\
\hline 28 & 200 & $\begin{array}{c}\text { Initiation of Warfarin } \\
\text { Discharge Home on 5 mg of } \\
\text { warfarin }\end{array}$ \\
\hline
\end{tabular}

\section{Case Discussion}

Heparin Induced Thrombocytopenia is a life threatening complication of exposure to heparin that results in thrombocytopenia and thrombosis [1]. It commonly occurs within 5-14 days of heparin exposure. It can also occur within 1 day of heparin exposure provided patients have been exposed to heparin in the previous 30-90 days. In rare cases it might be seen 2-3 weeks after cessation of heparin therapy (delayed onset thrombocytopenia). Exposure to unfractionated heparin is associated with 5 to 10 folds higher risk than low molecular weight heparin [1]. HIT is more commonly seen in surgical patients than in medical patients. Females are higher risk as compared to males. Patients exposed to heparin for thromboprophylaxis after orthopedic surgery have higher risk of HIT than patient exposed to heparin for cardiopulmonary bypass. The incidence of HIT is low in patient on chronic hemodialysis despite frequent heparin exposure during dialysis. Incidence of HIT is also found to be low (0.1\%) in pregnancy for unexplained reasons [1].

It has been postulated that heparin binds to PF4 antigens on platelets which causes a conformational change resulting in formation of a neo antigen. The neo antigen induces the production of IgG antibodies which bind to the heparin-PF4 structure to form a heaprin-PF4antibody complex. The complex can bind Fc receptors on the surface of platelets, neutrophils and monocytes and not only activate these cells but also lead to thrombin generation / release which ultimately leads to hypercoagulability, thrombosis and thrombocytopenia [2].

Thrombocytopenia is most common manifestation seen in $90 \%$ of patients [3]. The platelet drop is usually more than $50 \%$ from the baseline and platelet count less than $20,000 / \mathrm{ml}$ are rarely seen. Thrombosis is seen in $50 \%$ of the patients with HIT. Venous thrombosis is four times more common than arterial thrombosis. Venous thrombosis is commonly seen in leg veins, cardiac vessels and skin. Arterial thrombosis is seen in heart (Myocardial infarct), CNS (stroke), limbs (acute ischemic limb) and internal organs like kidneys (infarct) and intestine (mesenteric ischemia) [3]. Skin necrosis and anaphylaxis are other clinical manifestations. Bleeding from thrombocytopenia is rarely seen.

Diagnosis is based on clinical suspicion which is guided by $4 \mathrm{~T}$ score [4]. Diagnosis is confirmed by laboratory testing which include immunological and functional assays for HIT antibodies. ELISA for PF4-IgG has a high sensitivity (>95\%)and serotonin release assay has a high specificity (>90\%) [5]. If $4 \mathrm{~T}$ score indicates a moderate or high probability of HIT treatment should be initiated while waiting for the results of these more definitive laboratory tests.

Treatment involves cessation of all forms of heparin therapy including IV and subcutaneous heparin, heparin flushes and heparin coated catheters. Initiation of alternative non heparin anticoagulation is necessary even in the absence of thrombosis as the risk of having a thromboembolic episode considerably high [2,6]. If any evidence of an arterial or venous thromboembolic event is found, HITT (heparin induced thrombotic thrombocytopenia) is confirmed and anticoagulation is continued for 3-6 months. If no evidence of thrombosis is found duration of anticoagulation is controversial [6]. One study recommends continuing anticoagulation for at least one 
month after the platelet count has stabilized. Unless absolutely necessary all forms and doses of heparin should be avoided in future [6].

The post-operative risk of HIT after cardiac surgery is low $(0.6-2.0 \%)$. It is important to differentiate between thrombocytopenia in post-operative setting from HIT. In patients with HIT, platelet count usually drops in the first 2 to 4 days, then rebound into normal range or beyond and then decrease once again. The biphasic pattern of platelet recovery in post cardiac surgery setting remains a strong predictor of HIT. In our case the platelet count decreased after the CABG, gradually improved and then decreased again showing the biphasic pattern.HIT antibodies slowly fade away in about 50-90 days after last exposure to heparin. Although there is no anamnestic response patients with HIT should avoid heparin for life whether for therapeutic or prophylactic purposes [7].

This case teaches us that any patient with recent heparin exposure who presents with unexplained thrombocytopenia and thromboembolic episode should raise suspicion for delayed onset thrombocytopenia. A careful review of the history, physical exam and imaging studies guided by the $4 \mathrm{~T}$ score should be conducted to determine the probability of heparin induced thrombocytopenia. Treatment for heparin induced thrombocytopenia should be initiated without any delay and primarily involves avoiding heparin and promptly initiating alternative means of anticoagulation. It is important to rule out this entity before initiating any form of heparin therapy in order to avoid adverse consequences.

\section{Conclusion}

Heparin induced thrombocytopenia is a life threatening complication associated with heparin use. It usually occurs within 5-10 days of ongoing heparin exposure. In rare instances it may occur 1-3 weeks after cessation of heparin therapy. It is a clinical diagnosis guided by $4 \mathrm{~T}$ score and treatment involves cessation of heparin and alternative anticoagulation.

\section{Acknowledgements}

None.

\section{Statement of Competing Interests}

The authors do not have any statement of competing interests

\section{Abbreviation}

HIT = Heparin Induced Thrombocytopenia

DVT $=$ Deep vein thrombosis

HITT = heparin induced thrombotic thrombocytopenia

O.D= optimal density.

\section{References}

[1] Warkentin TE. Think of HIT. Hematology Am Soc Hematol Educ Program 2006:408-414.

[2] Alving BM. How I treat heparin-induced thrombocytopenia and thrombosis. Blood2003; 101(1):31-37.

[3] Warkentin TE. Clinical presentation of heparin-induced thrombocytopenia. Semin Hematol 1998;35 4 suppl:9-16.

[4] Crowther MA, Cook DJ, Albert M, et al. The 4Ts scoring system for heparin-induced thrombocytopenia in medical-surgical intensive care unit patients. J Crit Care2010;25(2):287-293.

[5] Denys B, Stove V, Philippe J, Devreese K. A clinical-laboratory approach contributing to a rapid and reliable diagnosis of heparininduced thrombocytopenia. Thromb Res2008;123(1):137-145.

[6] Lewis BE, Aranda C, Lewis M, Hoppensteadt D, Walenga JM, Fareed J. Unlike heparins newer oral anticoagulants do not interact with HIT antibodies and maybe useful in the long-term anticoagulant management of heparin compromised patients [abstracts]. Am Soc Hematol Educ Program 2011; 118(21).

[7] Potzsch B, Klovekorn WP, Madlener K. Use of heparin during cardiopulmonary bypass in patients with a history of heparininduced thrombocytopenia. N Engl J Med2000; 343(7):515 